\title{
Status Analysis of Training of Professional Talented in Medical Information Education
}

\author{
Yuanqiang Wang, Shangfu Hao, Xiao Zhang \\ School of information science and engineering, \\ Hebei North University, \\ ZhangJiaKou Hebei province, 075000, china \\ E-mail:hbnuwyq@126.com
}

\begin{abstract}
With the rapid development of digital medicine, health big data and medical Internet of things, the demand for the talents with stronger comprehensive ablility of information has become more highlighted. On the basis of status analysis, the paper finds that enrollment scale, curriculum structure, stabilization and sustainablity of scientific research and so on are the main restrictive factots in the training of professional talents in medical information education, which provides a reference for the evaluation sysytem construction of the follow-up talents trainging, and the course teaching reform.
\end{abstract}

Keywords-recombination and innovation; IT talents; medical information; status analysis; suggestions of teaching reform

It has become most pressing concern to deeply reform the ordinary teaching, medical \& health innovation and development in higher education, according to the administrative files:"National medium and long-term plan for education reform and development(2010-2020)", "The CPC Central Committee and State Council opinions on deepening the medical and health system reform", "General undergraduate colleges and universities setting regulations on professional" and "Recommendations on strengthening domestic innovative approach working" submitted to the state council by academician of Duzheng YE.

Today, it's coincided to the vigorous development opportunities in digital medical, health of big data, medical Internet of things to the domestic medical and health field, however, the key and bottleneck for information turn into knowledge economy is still the lack of recombination \& innovation medical information personnel. Consequently, in the period of medical information with characters of big data, it's obviously to the sharp contradictions and conflicts between supply and demand, one come from the university talents training, the other from the urgent need among of information collaboration, information integration and sharing information economy. In this case, each educator should try their best and with the sense of mission and responsibility to train the talents with charaters of compound, innovative and high-quality medical and health information professional knowledge.

\section{THE WHOLE OBJECTS OF TALENTS TRAINING IN MEDICAL INFORMATICS}

The file "University undergraduate catalog and professional presentation" issued by the ministry of education in
1998, objective of personnel cultivation has been identified to the major of information management \& information system (IMIS). But the objective is mainly for non-medical department, that is to say, the objective is given service to the economic information management but not medical information.

Medical informatic proposed in 1950s and formed the independent discipline in1970s, remarkable achievements have been achieved in Europe and American. But in domestic, it's the transformation of information science, library and archive science, information management science used in medical. Although the name of professional is not the same, such as: Medical Informatics, Medical Informatics Engineering. But the ultimate goal of training medical information talents satisfied with the hospital and medical \& health management section at all levels in national has not changed. Moreover, the academic qualifications from BA, $\mathrm{MA}, \mathrm{PhD}$ of medical education become more comprehensive in the key universities like Jilin universtiy, Central \& sourth university, Fudan university, Sichuan university etc. These institutions have made great achievements in medical informatics doctoral education and discipline construction, these achievements provides an important reference for other institutions of medical education and professional development.

Meanwhile, embarrassed with a wider range research but not in-depth have been found on medical information as a cross-disciplinary to medical, management and information technology, for the influence of incubation time relatively short, merger of colleges and enrollment expansionin on ,medical information talents training in higher education. So combined with the overall objectives of social development needs, the training goal on medical information professionals is to shape multivariate compound talents who can provide information technology support for the upper sector understanding, in the same time, with ability of basic theory of medicine, part of the management methods in business, takeing advantage of information technology as a tool and means to solve variety of medical affairs of information management, analysis, extraction and application.

\section{STATUS ANALYSIS OF TRAINING OF PROFESSIONAL TALENTED IN MEDICAL INFORMATION EDUCATION}

Based on the starting point of the overall goal of personnel training, research group made analysis to the status of domestic medical informatics talents training from four 
aspects: enrollment status, professional title structure, professional status and research status, according to the investigation data and charts.

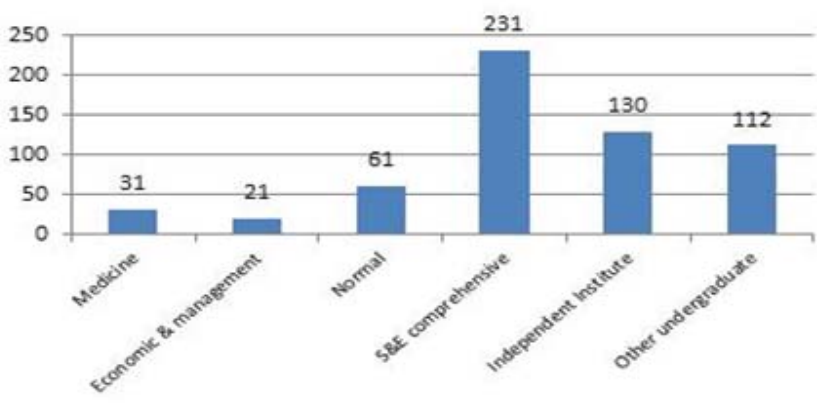

Figure 1. Distribution of information management profession

\section{A. Enrollment status of medical information professional}

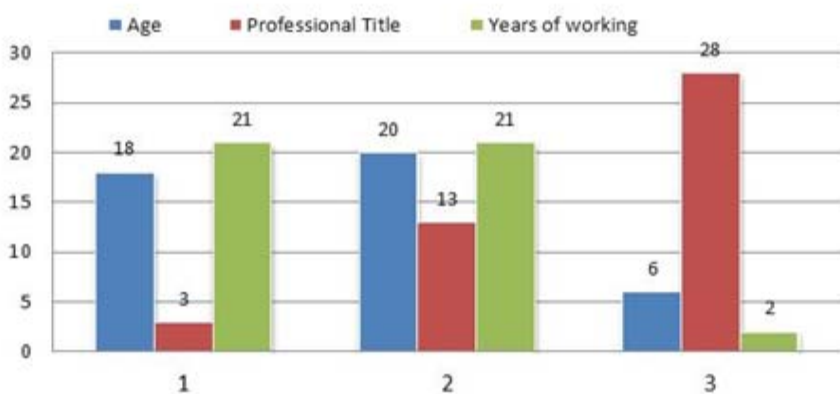

Figure 2. The chart for investigators's basic information

According to the file "The White Paper of Chinese Hospital Information Construction Survey” published in 2008, there are 3765 hospitals in domestic, but the ratio of IT talents to staff is under 0.5 percent in more than half of total hospitals. But in 2011, there are 1112 colleges and universities, only 586 enroll information management and information system majors. Especially, only 40 enroll the IMIS professional in 280 medical colleges and universities, 8 colleges eroll medical informatics, 4 colleges eroll meidical information engineerning. In the otherwise, the ratio of medical information personnel training institutions to ordinary institutions of higher learning is only 5 percent. As shown in Figure 1, the distribution of medical information professional was showned. The Fig. 1 reflects that the number of output of information talents from medical colleges in personnel training is hard to meeting the demand of growing national health.

\section{B. Status of professional title structure of medical informatics staff full-time teachers}

Currently, the most full-time teachers of medical informtics in institute is the profession transformaiton of medical, computer science, mathmatics, even some teacher is secondment from library and information science from economic management and so on. The result is the teaching task complited, but can’t grasp the integrity and develop- ment of the curriculum system, can't give reasonable proposal and opinions to the future development, learning focus and work-oriented of the professional student. Meantime, due to changes in school for teacher evaluation policy, attitude affected to full-time teachers who concentrated on positive discipline related areas subject to a certain extent, quick success, impetuous and so on became the main influence factors to professional healthy development and academic deepening reform.

According to the analysis of the questionnaire of 7th seminar of national medical informatics education for sustainable development, 60 questionnaires were issued and 44 valid questionnaires received, recovery rate is 73.3 percent. Status distribution about age, professional titles and working years were shown in Figure 2. Series of age from left to right expression the staff number of $(20,40),(40,50)$ and $(50,60)$. Series of profession title from left to right expression the staff number of early grades, secondary vocational and higher vocational. Series of working age expression the nubmer of 10 -years intervals seniority status. From the Fig.2, we found that the young teachers' professional title is lower, and the number of people committed to medical informatics education research is insufficient.

\section{Teaching status of medical informatics professional}

From the the questionnaire we found the investigator come from more than 40 institutes in different regions. Most of people are the school-related professional person in charge, academic leaders, doctoral, or master tutor. So we can say that the questionnaire basically reflects the professional problems in whole country. The questionnaire was conducted a preliminary design from 4 aspects and 25 related questions. The 4 aspects are the direction of professional training, recruitment and employment, curriculum and teachers construction. The shortcomings and deficiencies were found from the the analysis of the questionnaire results, that's in the medical informatics talents cultivation process. Direction in training: 47.73 percent think the curriculum lack of target in setting, 43.18 percent think current education mode is diffcult to meet with the needs of training objectives, 40.91 percent think the practice teaching is weak, and 38.64 percent think the professional social awareness is low. The common problems existed in training process was shown in Figure 3.

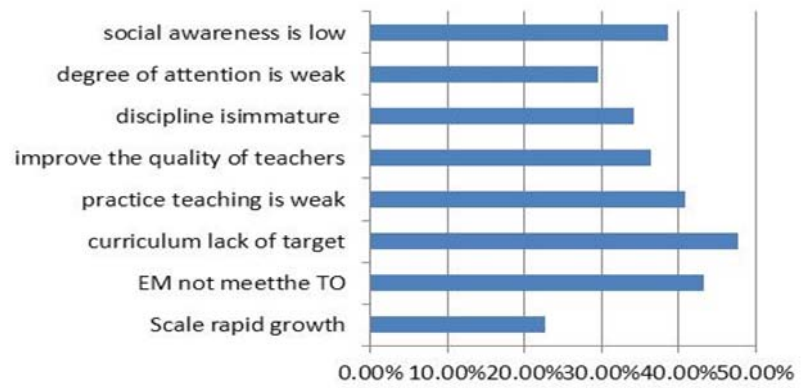

Figure 3. The problem existed in medical information education 
In Fig.4, some urgent problems were shown that need to be solved. In these questions, the most intense problems are solutions for professional orientation, curriculum and textbook construction. Following problem is the matching theory and social practice. That's exactly the embarrassing state to social enterprise faced in the recruitment process. Although, the school arrangement nearly a semester interships in hospital information management department, administrative departments and the medical \& health business to students, including the information management and health management graduate internships. But we found from statistical analysis datas which feedback by practice units that the students have a fairly high level of theory knowledge, while the actual operation ability is not strong in the concrete work of medical information management. Student's self-initiative is strong, but the observational and sensitivity is not strong to the progressive evolution law of between things. Student's interpersonal skills are excellent in working, but the standardization and seriousness are lacking in drafting application report and writing official documents. They familiar the using of computer, but not familiar the office application software developed for medical. There is no integrity and innovation in developing software of medical information system. Especially, they don't know how to better and standard handle the cash flow information in medical enterprise.

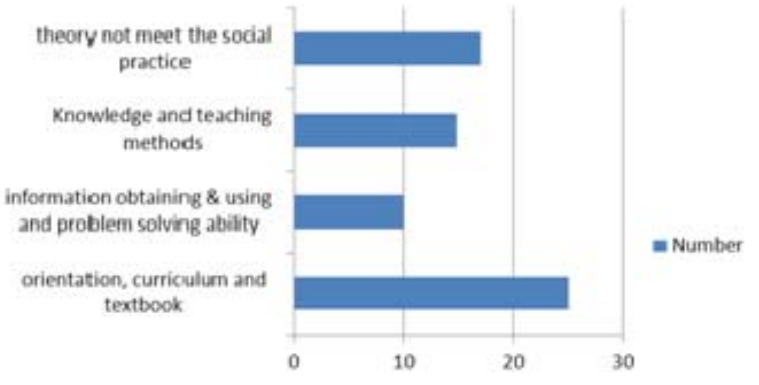

Figure 4. Some urgent problem were shown that need to be solved

In Fig. 5 the experts' consensus of professional development to medical informatics were shown. That's means, the courses of computer, mathematics \& statistics, medicine \& health should be strengthened in teaching of medical informatics professionals.

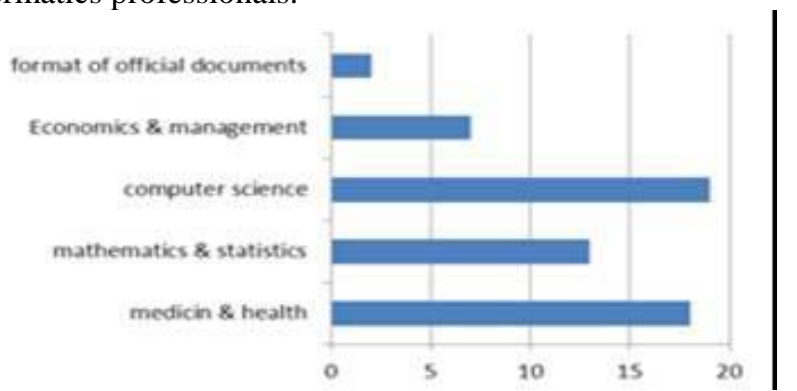

Figure 5. Strengten oriented to professional development

\section{Status of medical informatics research}

With using the web database resource of PubMed and CNKI which provieded by library of Hebei North University, by searching the subject words of "medical information" with English \& Chinese, retrieve the relevant academic papers 180314 in PubMed and 4125 in CNKI. In Figure 6, shown the situation at domestic and foreign papers published for the past 18 years between 1994 and 2012.

From the Fig.6, we found that the research of medical information showed steady growth mode in abroad, but relatively wavy motion in home. Maybe from side shown it impact can not be ignored that the reform of higher education to teaching \& science research for teachers.

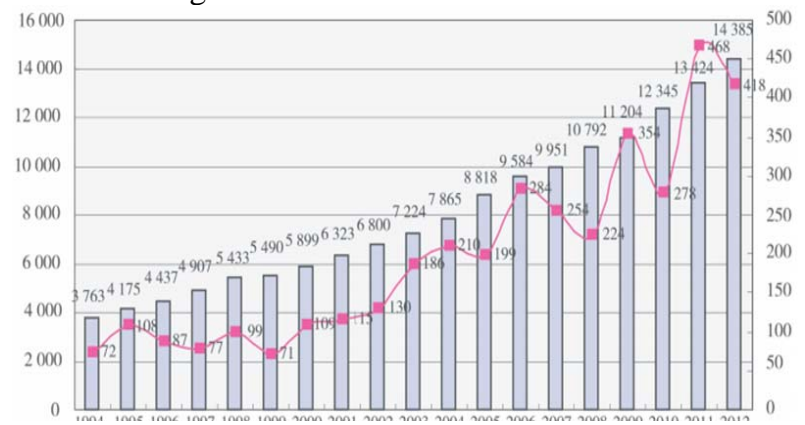

Figure 6. Annual statistics of papers at domestic and foreign in medical informatics

Especially, when we change the searching subject words from "medical information" to "medical informatics" in CNKI, the number of papers will sharply down form 4125 to 2316. Futhermore, major research institutions on medical informatics at dometic is institution of medical informaiton \& intelligence for each province, Central Sourth University, Shanxi Medical University, China Medical University, Jilin University and other comprehensive universities. For other institutions setting the professional of medical informatics, science research should be enhanced. Parts of paper quantity and institution ranking were shown in figure 7.

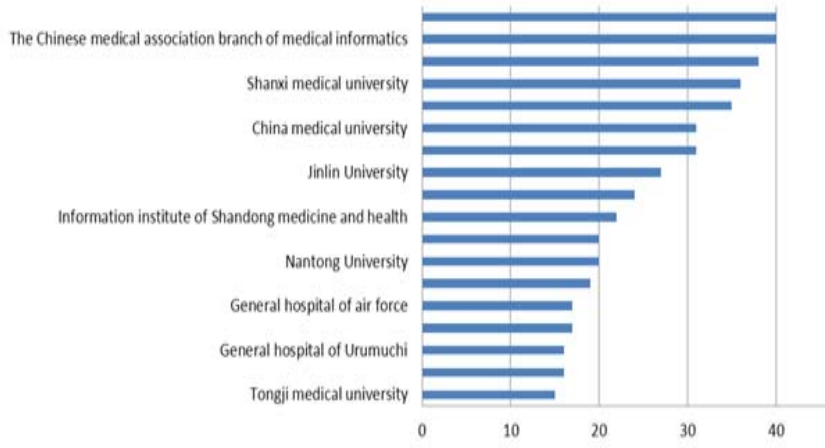

Figure 7. The paper distribution of major research institutions in medical information

In the future, with the development of information technology, health big data and IOT in health, we can believe the subjects of research and application is widely in the 
fields of medical informatics. For medical informatics teaching further research and development in domestic, the top 20 important phrases were selected to shown in Figure 8, which from the 180314 papers releated to the medical information.

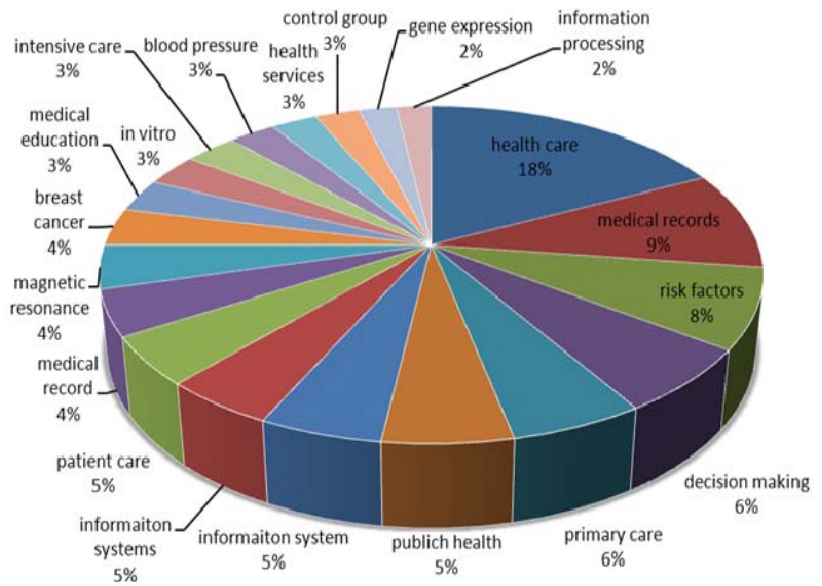

Figure 8. Top 20 important phrases relevant to medical informatics

\section{CONCLUSION AND ADVICE}

In the specific historical period, Medical informatics education will face many specific problems. But the most fundamental problem is still the development of the professional orientation, education theory and practice of teaching etc. Only the higher edcation, scientific research institutions, academic groups, social enterprises, government departments work together and intercommunication, moreover, the teaching method and curriculum system were innovated, we can truly make medical information education professional high positioning and targeted clearly, can meet the development of social factors: enterprise talent demand, to achieve the virtuous circle between talent training and the demand.

So in during of construction the talents training system for each institute and colleges, it can rely on the key disciplines broaden the undergraduate and graduate enrollment and size. They can formulate professional primary, intermediate and advanced courses system combined with the institutions of higher learning \& scientific research institutions which have the doctoral granting in medical informatics. Besides efforts to improve the teaching methods of professional teachers in this region, they can also vigorously promote useing the teaching resources network of national excellent course. In practice teaching activities, students should be encouraged to conduct practice in campus, scien- tific research and corporate practice, in order to achieve the combine of theory and practice. Simultaneously, researchers can do better in presonnel trainning and personalized service by take advantage of mobile Internet technology, digital medical technology, medical \& health IOT in the 3 rural medical health information field.

\section{ACKNOWLEDGEMENTS}

The authors would like to thank the research of comprehensive demonstration research of rural three-level health service, project number 2012BAJ18B08, which supported by the ministry of science and technology in china. At the same time, it's supported by HeBei north university (JG201310), science and technology department of Hebei province (112135119) and Higher education institute of Hebei province (gjxhz2011-25).

Corresponding author: Xiao Zhang, Professor.

\section{REFERENCES}

[1] Bethany S.D, Jethin R. Mobile Medial Education (MoMed)-how mobile information resources contribute to learning for undergraduate clinical students a mixed methods study. BMC Medical Educaiton, 2012, (12):1-11.

[2] Elena Melus-Palazon, Cruz Bartolome-Moreno.Experience with using second life for medical education in a family and community medicine education unit. BMC Medical Educaiton, 2012, (12):30-39.

[3] Fenghua Zhao. The apperception of Graduation practice teaching in Medical information management and information systems professional.The journal of medical informatics.2010, 20(54):54-56.

[4] Gaofang Cao, Weiwei Yu.The comparation of Medical information education research of domestic and overseas. Journal of Preventive Medicine Information, 2013, 29(1):62-66.

[5] Jiaming Bai.The investigation of construction of teacher's team in Medical information management and information systems professional.The journal of medical informatics .2010, 31 (5):87-89.

[6] Liwei Wang, Dongmei Mou, Wei Wang. The Enlightenment of Elearning to the domestic medical information education.China higher medical education.2010, (3):24-26.

[7] Shijing Zhang, Zhaoqin Hu. Status investigation and analysis of thedomestic medical information professional education.Chinese Journal of Medical Library and Information Science, 2006, 15(6):51-55.

[8] Wei Wang. The 25th anniversary summary and evaluation to medical informatics education in China. The journal of medical informatics.2011, 19 (11):4-7.

[9] Xiaodong Zhao, Junxia Zhai, Sugang Liu etc. Status research for the medical staff wtih the Internet for medical information belongs to Hebei province. Medical Information, 2010, 23 (1):92-93.

[10] Yanhua Lv, Qi Yu, Peifeng He. Papers on medical information science published in china: a bibliometric analysis. Chinese Journal of Medical Library and Information Science, 2013, 22(6):25-35.

[11] Yuanwu Zhang, Fen Zhang, Min Wang Etc. The investigation and study of information literacy to students in Medical colleges.The journal of medical informatics.2010, 31(9):83-87. 